\title{
A Study Comparing Patient and Clinician Perspectives of Treatments for Multiple Sclerosis via Group Concept Mapping
}

\author{
Barry A Singer (D) \\ Shannon Keith ${ }^{2}$ \\ Amy Howerter (D) $^{3}$ \\ Helen Doll (iD ${ }^{3}$ \\ Timothy Pham ${ }^{4}$ \\ Rina Mehta ${ }^{4}$ \\ IThe MS Center for Innovations in Care, \\ Missouri, Baptist Medical Center, \\ St. Louis, MO, USA; ${ }^{2}$ Clinical Outcomes \\ Assessment,Clinical Outcomes Solutions, \\ Chicago, IL, USA; ${ }^{3}$ Quantitative Science, \\ Clinical Outcomes Solutions, Tucson, AZ, \\ USA; ${ }^{4}$ US HEOR, Bristol Myers Squibb, \\ Princeton, NJ, USA
}

Background: Clinicians treating multiple sclerosis (MS) should consider patient preferences when making treatment decisions. An online mixed-methods approach to elicit patientcentered concepts, group concept mapping (GCM), was used to generate statements reflecting the patient experience in relapsing-remitting MS and identify the most important patient-centered outcomes from patient and clinician perspectives.

Patients and Methods: Twenty patients and $12 \mathrm{MS}$ specialists in the United States provided statements describing what an ideal treatment would do to improve symptoms and daily functioning. Statements were sorted by participants into meaningful domains and rated on importance on an 11-point scale.

Results: Sixty-four unique statements supporting 6 domains of clustered concepts were generated. Patient and clinician ratings of importance were highly correlated $(r=0.82)$; however, patients rated the domains of Activities of Daily Living, Prevent \& Cure, and Address Symptoms as highest in importance, whereas clinicians rated Prevent \& Cure, Safe $\&$ Effective, and Activities of Daily Living as highest in importance. Statements rated above the domain mean by both patients and clinicians included "Improve cognitive function" and "Improve motor function" in the Activities of Daily Living domain and "Help with memory issues" and "Help preserve cognition" in the Address Symptoms domain. The statement "Improve short term memory" was 1 of 3 statements rated above the domain mean by patients but below the domain mean by clinicians.

Conclusion: High levels of agreement of concept importance were found between patients and MS specialists, although certain domains and statements were rated more highly by one group. Overall, concepts such as cognitive function, physical and emotional functioning, and activities of daily living were perceived as having great importance for treatment outcomes versus symptom-focused outcomes like gait or tingling sensations. This comprehensive concept model for the MS patient experience can be used for further development of patientcentered outcome measures in MS treatment.

Keywords: relapsing-remitting multiple sclerosis, patient outcomes, cognitive function, quality of life

\section{Plain Language Summary}

When evaluating treatments in clinical practice or clinical studies, assessment tools that gather input from patients and clinicians are essential, especially when evaluating more subjective outcomes such as effects on physical or emotional functioning or quality of life. The purpose of the current study was to explore the desired outcomes of treatments for multiple sclerosis (MS) using a mixed-methods approach. The study design used patients'
Clinical Outcome Assessments, Clinical Outcomes Solutions, $53 \mathrm{~W}$ Jackson Blvd, Suite II50, Chicago, IL, 60604, USA

Tel +I 3124652443

Email shannon.keith@clinoutsolutions. com 
and clinicians' own words to describe treatment-related concepts that are important to daily functioning in patients living with MS rather than predetermined treatment characteristics.

Results showed that although patients and clinicians described concepts of importance similarly, they did not necessarily rate them at the same levels of importance. Patients tended to rate concepts that affect their daily lives higher than concepts related to treatment safety or effectiveness. Both patients and clinicians rated cognitive impacts of MS as more important than some motor- or fatigue-related symptoms and impacts.

When considering the array of available MS treatment options that target various aspects of the disease, clinicians should consider patient preferences when selecting from among them. Additionally, patient-identified impacts on their daily lives should be considered for inclusion as outcomes in clinical studies testing treatment effectiveness.

\section{Introduction}

Multiple sclerosis (MS) is a chronic disease in which the immune system attacks the central nervous system (CNS), resulting in demyelination. ${ }^{1} \mathrm{MS}$ is classified into 3 subtypes: relapsing-remitting MS (RRMS), secondary progressive MS, and primary progressive MS. RRMS has been characterized by relapses followed by periods of partial or full recovery. The cause of MS is not entirely clear, although research suggests that it is a combination of various genetic and environmental factors. Women are affected at approximately 2 to 3 times the rate of that among men. ${ }^{1-3}$ Onset of MS usually occurs between 20 and 40 years of age, ${ }^{1}$ and, in a 2017 study, it was estimated to affect between 850,000 and 913,000 people in the United States. ${ }^{3,4}$ People with MS have a variety of symptoms owing to inflammation in the CNS; symptoms include fatigue, depression, pain, muscle tightness, tremor, gait impairment, memory loss, sexual dysfunction, and bowel and urinary tract issues. ${ }^{4}$

Currently, the treatment landscape for RRMS can be divided into 2 categories: (1) treatment targeted to specific symptoms and (2) disease-modifying treatment. In the past 20 years, numerous treatments in the latter category have become available to MS patients, thus increasing the importance of understanding what outcomes a patient may be seeking from a treatment. ${ }^{5}$ Conceptual models for MS identify fatigue, pain, musculoskeletal issues such as stiffness and spasm, and balance problems as key symptoms and impacts of MS. ${ }^{6}$ When comparing patient and clinician treatment goals using cognitive mapping, Col et $\mathrm{al}^{7}$ identified domains and preference attributes such as brain health, preventative measures, and daily living and provided insight into patient expectations for treatment outcomes beyond key symptoms such as fatigue and pain. There is a need for additional qualitative research on the patient perspective in MS, particularly among those with RRMS, to allow comprehensive measurement of MS symptoms and their impact on patients. ${ }^{8,9}$

The purpose of this study was to generate a better understanding of ideal treatment outcomes for RRMS by identifying the most important, relevant, and patientcentered treatment outcomes from both patient and clinician perspectives. This study used a novel mixed-methods approach, group concept mapping (GCM), which combines qualitative and quantitative methods to generate insights on key concepts and priorities. The outcome of the GCM process is to have concepts generated and grouped directly by patients and clinicians, with little interference from or interaction with researchers, to provide a conceptual model of outcomes that describes the potential impacts of treatments for MS and focuses on issues of management and care regarding patient day-today function (eg, cognition, physical function).

One particular feature of GCM is that it allows participants to take part in study activities online and provide spontaneous responses to a prompt question on a community interface where they can see other participants' responses, similar to an online focus group. Once all statements are received, the research team compiles the full list, removes duplicates and, if necessary, corrects spelling or grammar. In a second round of study activity, participants group and rate all of the statements on their own to capture how participants themselves organize the ideas generated. Quantitative analysis of the sorting and rating results, using multidimensional scaling (MDS) and hierarchical cluster analysis (HCA), produces a shared framework that is informed by both patients and clinicians.

GCM is an alternative method to existing extensive interviewing and code-based qualitative analysis, ${ }^{10}$ which can be time-consuming and, notably, may be influenced by the investigator's own objectives. Using GCM, we present a framework of ideal outcomes for RRMS treatments that can be used to support and inform other conceptual models of MS outcomes and for the development of future patientreported outcome (PRO) scale development. ${ }^{11}$

\section{Materials and Methods \\ Study Design}

The GCM research method included 3 data collection activities or tasks (Figure 1). 


\section{GCM Collection Activities}

\begin{tabular}{|c|c|c|}
\hline $\begin{array}{c}\text { Task 1: } \\
\text { Concept Generation }\end{array}$ & $\begin{array}{l}\text { Task 2: } \\
\text { Sorting }\end{array}$ & $\begin{array}{l}\text { Task 3: } \\
\text { Rating }\end{array}$ \\
\hline $\begin{array}{l}\text { Participants responded } \\
\text { to the prompt: } \\
\text { "An ideal treatment to treat the } \\
\text { symptoms of multiple sclerosis and } \\
\text { impact my day-to-day function (eg, } \\
\text { physical, cognitive) would..." }\end{array}$ & $\begin{array}{l}\text { Statements were sorted into } \\
\text { groups created by the participant }\end{array}$ & $\begin{array}{c}\text { Statements were each rated } \\
\text { in terms of importance from } \\
0 \text { (not important) to } \\
10 \text { (extremely important) }\end{array}$ \\
\hline $\begin{array}{l}\text { Patients } n=20 \\
\text { Clinicians } n=11 \\
\text { Statements } n=99\end{array}$ & $\begin{array}{l}\text { Patients } n=18 \\
\text { Clinicians } n=12 \\
\text { Statements } n=64\end{array}$ & $\begin{array}{l}\text { Patients } n=18 \\
\text { Clinicians } n=12 \\
\text { Statements } n=64\end{array}$ \\
\hline
\end{tabular}

Figure I GCM task flow.

Abbreviation: GCM, group concept mapping.

Task 1, Concept Generation: Using an online portal called GroupWisdom, clinicians and patients separately generated unique statements that reflected their perspective on what would be ideal treatment aspects for MS. Upon accessing the GCM online platform, participants were presented with basic instructions and the following focus prompt to consider: "An ideal treatment to treat the symptoms of multiple sclerosis and impact my [patients'] day-to-day function (eg, physical, cognitive) would ...." Participants were instructed to type in as many individual responses to the prompt as desired. For 10 days, participants could log into the GCM platform at any time to enter statements and see what other participants had entered. Researchers then reviewed all statements in a harmonization process to reconcile any duplicates, spelling or grammatical errors, and other irregularities.

Task 2, Sorting: Following harmonization, a combined final list of statements was uploaded on the GroupWisdom platform and a second web link was sent to all participants. Clinicians and patients were instructed to sort the list of statements generated from Task 1 brainstorming into categories. Instructions were to " ... sort the statements into different groups. We want you to group them based on how you believe they are similar or related to one another." Participants were instructed to make as many or as few groups as desired and to label their groups with "a new name that reflects your idea of how the contents of the pile are similar or related." A group was identified as at least 1 set of items being paired together. Participants could leave statements ungrouped if they thought the statements did not belong with any others.

Task 3, Rating: Following sorting, all participants individually rated each statement on importance for the overall treatment of MS on a numeric rating scale (NRS) anchored with 0-Not Important at All to 10-Extremely Important. The importance rating question was, "Please rate all of the statements listed below based on the importance of each for the overall treatment of multiple sclerosis." The 11point importance ratings were done to aid the comparison of domains in the final concept map.

\section{Study Population}

Patients were recruited through the use of a recruitment agency from local clinician offices (ie, neurology centers treating RRMS patients) in 4 US cities: Baltimore, New Orleans, Chicago, and St. Louis. The recruiters and site staff introduced the study to potential participants, obtained written consent, and collected demographic data. A patient with RRMS was considered eligible for participation in the study if they met the following criteria: RRMS diagnosis; ambulatory; aged $\geq 18$ years and $\leq 55$ years; English language speaking; access to the internet; and provision of written informed consent. Patients were ineligible if they had conditions that might interfere with participation (ie, physical condition that would prevent completion of questionnaires) and if they were diagnosed with a nonrelapsing form of MS. The demographic characteristics of the sample are provided in Table 1.

MS specialists who participated in the GCM tasks were recruited separately by email invitation through the use of a recruitment agency. Participating clinicians were from Florida, North Dakota, Baltimore, New Orleans, Chicago, and Los Angeles. A clinician was considered eligible for participation in the study if they met the following criteria: licensed medical doctor (MD) or osteopath (DO) with board certification in neurology; current neurology practice within the US; and provision of verbal informed consent. Clinicians were ineligible if they had a condition that 
Table I Demographic Information for MS Patients

\begin{tabular}{|c|c|c|}
\hline $\begin{array}{l}\text { Demographic } \\
\text { Variables }\end{array}$ & Statistics & $\mathbf{N}=\mathbf{2 0}$ \\
\hline \multirow[t]{4}{*}{ Age, y } & $\mathrm{n}$ & 20 \\
\hline & Mean (SD) & $44.3(10.5)$ \\
\hline & Median & 42.0 \\
\hline & Min, $\max$ & 25,66 \\
\hline \multirow[t]{2}{*}{ Sex, n (\%) } & Female & $15(75.0)$ \\
\hline & Male & $5(25.0)$ \\
\hline Ethnicity, n (\%) & Not Hispanic & $20(100)$ \\
\hline \multirow[t]{2}{*}{ Race, n (\%) } & White & $15(75.0)$ \\
\hline & Black/African American & $5(25.0)$ \\
\hline \multirow[t]{5}{*}{ Education, n (\%) } & Did not complete high school & I (5.0) \\
\hline & High school diploma & $2(10.0)$ \\
\hline & $\begin{array}{l}\text { Some college or certification } \\
\text { program }\end{array}$ & $6(30.0)$ \\
\hline & College or university degree & $8(40.0)$ \\
\hline & Graduate degree & $3(I 5.0)$ \\
\hline \multirow[t]{5}{*}{ Work status, n (\%) } & Employed full-time & $14(70.0)$ \\
\hline & Employed part-time & $2(10.0)$ \\
\hline & Homemaker & $2(10.0)$ \\
\hline & Retired & I (5.0) \\
\hline & Other & I (5.0) \\
\hline
\end{tabular}

Abbreviation: MS, multiple sclerosis.

might interfere with participation (ie, physical condition that would prevent completion of questionnaires). Background information of recruited clinicians is provided in Table 2.

All study procedures were approved by an institutional review board (IRB) (Western Institutional Review Board; protocol \#HEOR-MS-CG1024C). Participant informed consent (written or verbal) was obtained as approved by the IRB.

\section{Data Analysis}

A Concept Systems facilitator (Scott Rosas) analyzed the sorting and rating data using GCM software (Concept Systems Inc., Albany, OR, USA). ${ }^{12}$ Figure 2 displays the GCM analysis steps. A similarity matrix, MDS, and HCA were used to depict relationships between statements,
Table 2 Background Information for Participating MS Specialists (Clinicians)

\begin{tabular}{|l|c|}
\hline Clinician Background Characteristic & Total (N = I2) \\
\hline Years qualified as MD, $\mathrm{n}$ & $\mathrm{I}$ \\
6-10 years & $\mathrm{I}$ \\
II-20 years & 10 \\
More than 20 years & \\
\hline Type of practice, $\mathrm{n}$ & 12 \\
Private practice & \\
\hline Current type of patient population, $\mathrm{n}$ & 5 \\
Outpatient only & 7 \\
Combination of inpatient and outpatient & 1 \\
\hline Years treating MS, $\mathrm{n}$ & $\mathrm{I}$ \\
6-I0 years & 10 \\
II-20 years & \\
>20 years & $6 \mathrm{I} \mathrm{(49.5)}$ \\
\hline MS patients clinic manages per month (average), $\mathrm{n}$ & $10-150$ \\
Mean (SD) & \\
Min-max & $92(4.1)$ \\
\hline Patients currently on treatment, \%, mean (SD) & $7.1(4.8)$ \\
Disease-modifying therapies & \\
Symptomatic management therapies & \\
Not prescribed any therapies & \\
\hline
\end{tabular}

Abbreviations: MD, medical doctor; MS, multiple sclerosis.

create the final clusters using the grouping information provided by the participants, and generate point, cluster, and cluster rating concepts maps, ${ }^{13,14}$ as described below and shown in Figure 2. Pattern-matching comparisons of cluster ratings and scatter plots were created to examine statement ratings within each domain.

\section{Point Map}

Based on participants' sorting data, the point map was created using a similarity matrix, which calculated the frequency of participants' grouping of each symptom/ impact concept in the list with another symptom/impact concept (ie, placed 2 concepts into the same pile). The closer 2 points are to each other, the more often participants sorted these statements together. Fit statistics of the point maps were assessed to ensure adequate fit between the group-level point map and sorting data, as indicated by the "stress value."13 The stress value is a measure of how similar the distances between points on the point map are to the distance of the values in the input similarity matrix and thus is a rough indicator of the degree to which the maps represent the sorting data. Although Kane and 


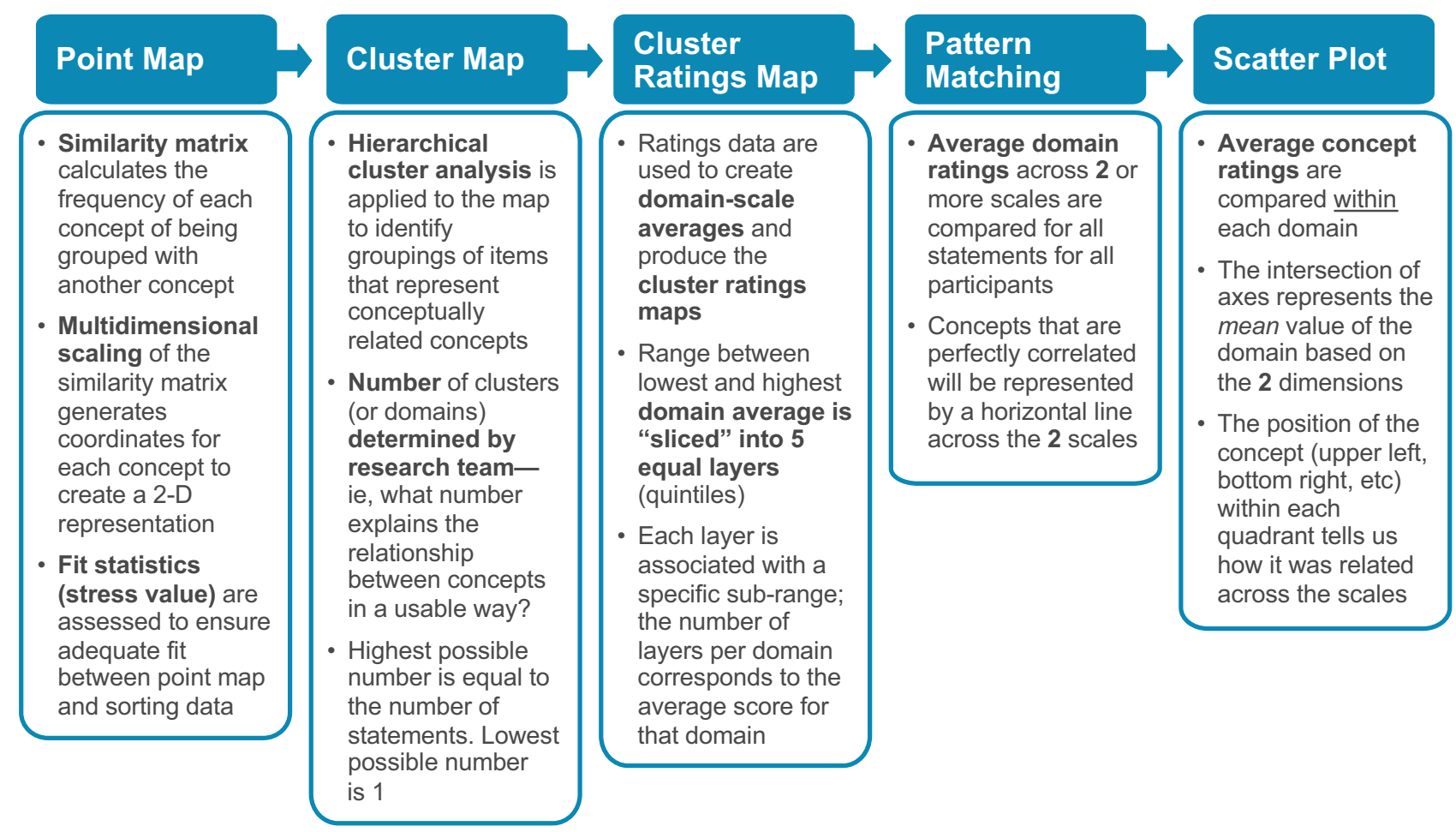

Figure 2 GCM analytic steps.

Abbreviations: GCM, group concept mapping, 2-D, 2-dimensional.

Trochim $^{13}$ suggest using a stress value of 0.285 as a threshold for concept maps (based on unpublished metaanalyses they performed on published papers of concept mapping projects), a lower stress value of 0.15 is a criterion more widely accepted by MDS experts. ${ }^{15}$

\section{Cluster Map}

Cluster maps were created to display each of the points as 2-dimensional shapes based on how conceptually similar or dissimilar statements were to each other. The similarity matrix and MDS were followed by an HCA on the Euclidean distances between statements, as plotted on the resulting 2-dimensional MDS map (Figure 2). The number of clusters (or domains) for each map was determined by the research team after considering the largest and smallest number of clusters that explained the relationship between concepts in a usable way. The largest possible number of clusters is always equal to the number of statements in the map, and the smallest possible number of clusters is 1 . The analytic decision-making involved in creating the best fit map involved iterative reviews of the model, seeking a solution that generated the lowest stress value possible for the point map along with defined boundaries between clusters for the cluster map. The domain names were determined by studying the individual statements within each domain, as well as the cluster labels provided by participants, and research team agreement on a name that best represented all statements within the domain.

Domain scale averages were generated using the importance rating data for the items within the domain, which were used to create the cluster ratings maps. The layers of the cluster ratings map are a visual representation of the range of the domain averages, with each layer representing a quintile of the total range. Thus, a domain with 5 layers indicated that the domain average fell in the upper quintile of the total range of ratings and was rated higher in importance overall than a domain with 1 layer (the lower quintile of the importance range).

\section{Pattern Matching}

Pattern matching comparison of the domain ratings of the 2 groups (clinicians vs patients) was performed. The pattern matching correlation coefficient is a Pearson $r$ correlation between the average ratings of 2 variables, in this case the patient and clinician scale ratings on importance. A positive cluster-level correlation between the patients and clinicians would suggest that each group views the most important concepts of MS treatment 
outcomes in a similar pattern. A negative correlation would indicate an inverse relationship on clusters of importance between patients and clinicians.

\section{Scatter Plots}

Scatter plots were created to show the relative ratings of statements within each domain, allowing analysis at the item level (as opposed to the domain-level analysis presented in the pattern matching). The mean ratings of each statement were placed in a graph of $x-y$ coordinates, with 1 variable on the $\mathrm{x}$ axis and another on the $\mathrm{y}$ axis. The graph is separated into 4 quadrants that are divided using the mean rating of the statements within the domain for the patients and clinicians. Statement ratings were thus grouped in terms of whether they are or are not above the average rating of that domain. Statements that were rated similarly (high for patients and clinicians) versus differently (for example, higher for patients and lower for clinicians) could be examined. The scatter plot diagrams were useful for determining which items within a domain to consider for deletion during ongoing measurement development.

\section{Results}

This study enrolled 32 participants (12 clinicians [all MS specialists] and 20 patients). Figure 1 displays the GCM study flow and participation. Participants could opt to complete some or all of the GCM activities. Eleven clinicians and 20 patients $(\mathrm{N}=31)$ completed the Concept Generation task; 1 additional clinician logged in but did not add statements. All 12 clinicians and 18 of the 20 patients (overall $\mathrm{n}=30$ ) participated in the Sorting and Rating tasks.

\section{Concept Generation}

For the Concept Generation task, participants $(n=31)$ generated 99 responses to the focus prompt (Figure 1). The process of harmonization led the research team to retain 64 statements uploaded to the GroupWisdom platform. The final list of statements used for sorting is shown in Table 3. The item number represents the order the statement was entered into GroupWisdom and presented for ratings.

\section{Point and Cluster Maps}

Sorting data were collated using the similarity matrix and MDS (Figure 3A). Fit statistics of the map were assessed to ensure adequate fit between the group-level point map and sorting data. The stress value was 0.2176 for the model, which is below the prespecified threshold of 0.285 and reflects a good fit between the point map and the similarity matrix (although it is higher than the more widely accepted MDS threshold of 0.15). The conceptlevel HCA to aggregate proximal concepts into domains based on their conceptual relatedness led to a 6-cluster group, or domain, solution shown in the point cluster map (Figure 3A). Although all 30 participants sorted statements, sorting data from 4 participants was excluded for the model to resolve into distinct clusters (ie, no overlap in shapes). Key reasons for exclusion were using a "miscellaneous" pile and 3 or fewer piles created. The cluster map is based on the point map, with all the points at the same coordinates as in the point map (Figure 3A). The individual statements that correspond to each point are listed (item numbers are reflected in Table 3). The cluster names were derived from the group names provided by the participants after the analysis determined which statements best fit in each cluster. Thus, the cluster names are simply what seemed to best represent the statements included in the cluster without changing any statements in the cluster. The 6 clusters include: (1) Activities of Daily Living (11 items), (2) Address Symptoms (13 items), (3) Medicine Assets (14 items), (4) Safe \& Effective (9 items), (5) Prevent \& Cure (9 items), and (6) Nonspecific (Holistic $\&$ Economical) (8 items). The statements in Cluster 6, Nonspecific (Holistic \& Economical), are not as similar in concept to one another compared with statements within Clusters 1 through 5 and take on characteristics related to both seeking alternative therapies or holistic patient approaches, as well as insurance reimbursement or coverage. This cluster was closely examined for division, assuming the statements could be divided into two. However, no model would fit well with these concepts separated, thus a final decision was made to keep this as a unified domain and label it as Nonspecific (Holistic \& Economical).

\section{Ratings}

The importance rating question was completed by 30 participants (12 clinicians and 18 patients). The importance ratings were analyzed to provide an average domain rating on which the importance cluster ratings map is based (Figure 3B). A domain with 1 layer was rated on average between 7.43 and 7.74 , while a domain with 5 layers was rated on average between 8.67 and 8.98. It is notable that the lowest cluster average was 7.43 , indicating that the ratings for all the domains were relatively high for 
Table 3 Descriptive Statistics for GCM-Derived Domains and Corresponding Statements

\begin{tabular}{|c|c|c|c|c|}
\hline Item ID ${ }^{\mathbf{a}}$ & Statements & Mean (SD) ${ }^{b}$ & Median & Min-Max \\
\hline \multicolumn{2}{|c|}{ Activities of Daily Living } & $8.63(2.23)$ & 9 & $0-10$ \\
\hline 3 & Improve motor function & $9.25(1.19)$ & 10 & $5-10$ \\
\hline 42 & Improve balance & $9.08(1.39)$ & 10 & $5-10$ \\
\hline 22 & Help physical function & $9.00(1.17)$ & 9 & $6-10$ \\
\hline 43 & Improve cognitive function & $8.97(1.35)$ & 10 & $5-10$ \\
\hline 2 & Help physical daily functioning for a more active social life & $8.69(1.37)$ & 9 & $5-10$ \\
\hline 5 & Help with visual impairment & $8.63(1.53)$ & 9 & $5-10$ \\
\hline 34 & Improve ambulation (walking) conveniently without harmful side effects & $8.59(1.58)$ & 9 & $5-10$ \\
\hline 56 & Address fatigue & $8.52(1.51)$ & 9 & $5-10$ \\
\hline 59 & Provide more sustained energy to return to high functioning & $8.50(1.6)$ & 9 & $5-10$ \\
\hline 55 & Focus on physical activity & $7.94(2.4)$ & 9 & $0-10$ \\
\hline 57 & Help improve gait & $7.87(2.75)$ & 9 & $0-10$ \\
\hline \multicolumn{2}{|c|}{ Address Symptoms } & $8.42(1.87)$ & 9 & $0-10$ \\
\hline 28 & Help with memory issues & $9.07(1.04)$ & 9 & $7-10$ \\
\hline 64 & Treat pain associated with multiple sclerosis (MS) conveniently and safely & $9.00(1.47)$ & 10 & $5-10$ \\
\hline 18 & Help preserve cognition & $8.87(1.31)$ & 9 & $5-10$ \\
\hline 16 & Restore sensation or feeling & $8.73(1.37)$ & 9 & $5-10$ \\
\hline 35 & Improve short term memory & $8.66(1.45)$ & 9 & $5-10$ \\
\hline 20 & Relieve the symptoms to make one's daily routine easier & $8.59(1.38)$ & 9 & $6-10$ \\
\hline 40 & Effectively treat bladder problems & $8.45(1.75)$ & 9 & $4-10$ \\
\hline 53 & Provide a cure for tingling & $8.36(2.27)$ & 9 & $1-10$ \\
\hline 54 & Treat spasticity (stiffness, tightness, cramping, spasms) with medication & $8.30(1.86)$ & 9 & $3-10$ \\
\hline 45 & Be one that patients notice an improvement in their function when taking it & $8.23(2.3)$ & 9 & $2-10$ \\
\hline 60 & Include dizziness treatment to reduce spinning & $8.10(1.63)$ & 8 & $5-10$ \\
\hline 49 & Take away shaking & $8.04(2.35)$ & 9 & $0-10$ \\
\hline 29 & Include treatment for insomnia & $7.11(2.85)$ & 8 & $0-10$ \\
\hline \multicolumn{2}{|c|}{ Medicine Assets } & $7.71(2.64)$ & 9 & $0-10$ \\
\hline 31 & Be a medicine that would slow the progression of the disease & $9.35(1.05)$ & 10 & $7-10$ \\
\hline 7 & Be a medication with no side effects & $9.21(1.24)$ & 10 & $5-10$ \\
\hline 27 & Have no or minimal side effects & $8.97(1.38)$ & 10 & $6-10$ \\
\hline 17 & Be a medicine that would allow one to lead a normal, healthy lifestyle & $8.90(1.30)$ & 10 & $6-10$ \\
\hline 14 & $\begin{array}{l}\text { Be a once-a-day medication with no risk of progressive multifocal leukoencephalopathy } \\
\text { (PML) or cancer }\end{array}$ & $8.80(1.38)$ & 9 & $5-10$ \\
\hline 36 & Effective and safe drug for fatigue & $8.52(1.48)$ & 9 & $4-10$ \\
\hline II & Be one medication to address all symptoms & $8.14(1.91)$ & 9 & $2-10$ \\
\hline 6 & Include a "booster" taken in flare-up situations to limit the negative impact on the body & $7.90(2.34)$ & 8 & $0-10$ \\
\hline 52 & Be an alternative to injections & $7.84(2.47)$ & 8 & $1-10$ \\
\hline 24 & Be a medication taken once a month or longer & $7.52(2.37)$ & 8 & $0-10$ \\
\hline 38 & Treat pain with medication without sedation & $7.25(2.48)$ & 7 & $0-10$ \\
\hline 50 & Not require refrigeration & $5.50(3.08)$ & 6 & $0-10$ \\
\hline 26 & Be a liquid medication & $5.25(3.49)$ & 6 & $0-10$ \\
\hline 37 & Be medication in the form of a gummy & $4.83(3.38)$ & 5 & $0-10$ \\
\hline \multicolumn{2}{|c|}{ Safe \& Effective } & $8.31(2.23)$ & 9 & $0-10$ \\
\hline 48 & Be safe, convenient, and reverse previous damage & $9.14(1.36)$ & 10 & $5-10$ \\
\hline 30 & Increase percent of patients who are relapse free & $8.69(1.42)$ & 9 & $5-10$ \\
\hline 46 & Work without putting the body at risk of cancer or heart problems & $8.63(2.36)$ & 10 & $0-10$ \\
\hline
\end{tabular}

(Continued) 
Table 3 (Continued).

\begin{tabular}{|c|c|c|c|c|}
\hline Item ID ${ }^{\mathbf{a}}$ & Statements & Mean (SD) ${ }^{b}$ & Median & Min-Max \\
\hline 62 & $\begin{array}{l}\text { Have no increased chance of malignancy, low white blood cell count, or increase risks of } \\
\text { infection }\end{array}$ & $8.57(2.45)$ & 10 & $0-10$ \\
\hline 33 & Be one where the effectiveness does not decline over years & $8.52(1.5 I)$ & 9 & $5-10$ \\
\hline 19 & Be a cure for progressive multifocal leukoencephalopathy (PML) & $8.45(2.27)$ & 9 & $0-10$ \\
\hline 51 & Include information on long term side effects and safety & $7.90(2.87)$ & 9 & $0-10$ \\
\hline 32 & Require no monitoring & $7.68(2.08)$ & 8 & $2-10$ \\
\hline 44 & Provide data on different types of medications & $7.18(2.83)$ & 8 & $0-10$ \\
\hline \multicolumn{2}{|c|}{ Prevent \& Cure } & $8.98(1.90)$ & 9 & $0-10$ \\
\hline 41 & Reverse prior deficits & $9.32(0.93)$ & 10 & $7-10$ \\
\hline 25 & Stabilize progression while reversing damage that has occurred in the brain & $9.25(1.49)$ & 10 & $3-10$ \\
\hline 4 & Focus on a permanent solution for all kinds of multiple sclerosis (MS) & $9.22(1.38)$ & 10 & $5-10$ \\
\hline 47 & Protect nerve cells and aid in neural repair & $9.20(1.30)$ & 10 & $5-10$ \\
\hline 63 & Include a way to detect progression before harm is caused & $8.97(2.03)$ & 10 & $0-10$ \\
\hline I & Be a vaccine to prevent the disease & $8.94(2.09)$ & 10 & $0-10$ \\
\hline 10 & Be a cure for multiple sclerosis (MS) & $8.90(2.70)$ & 10 & $0-10$ \\
\hline 39 & Make one multiple sclerosis (MS) free & $8.75(2.31)$ & 10 & $0-10$ \\
\hline 15 & Include a rapid blood test to monitor and direct the right treatment & $8.32(2.27)$ & 9 & $0-10$ \\
\hline \multicolumn{2}{|c|}{ Nonspecific (Holistic \& Economical) } & $7.43(2.74)$ & 9 & $0-10$ \\
\hline 23 & $\begin{array}{l}\text { Have insurance approve medication of your choice without having to fail two prior } \\
\text { medications }\end{array}$ & $8.80(2.13)$ & 10 & $0-10$ \\
\hline 13 & Be inexpensive & $8.52(2.31)$ & 9 & $0-10$ \\
\hline 21 & Take the entire patient into account & $8.14(2.28)$ & 9 & $0-10$ \\
\hline 58 & Lead to feeling better about myself & $7.40(2.61)$ & 8 & $0-10$ \\
\hline 12 & $\begin{array}{l}\text { Have insurance coverage for holistic and alternative treatments such as yoga, massage, } \\
\text { acupuncture }\end{array}$ & $6.97(2.62)$ & 7 & $0-10$ \\
\hline 9 & Focus on dietary changes that improve immune function & $6.87(2.73)$ & 8 & $0-10$ \\
\hline 61 & $\begin{array}{l}\text { Include information for patients to know when they should contact their primary doctor } \\
\text { instead of their MS doctor }\end{array}$ & $6.50(2.96)$ & 8 & $0-10$ \\
\hline 8 & Be an advanced diet & $6.25(3.30)$ & 7 & $0-10$ \\
\hline
\end{tabular}

Notes: ${ }^{a}$ Items sorted by their mean importance rating (largest to smallest) within each domain. ${ }^{b}$ Domain mean and standard deviation are calculated by averaging responses from all participants across all items within the domain. Item mean and standard deviation are calculated by averaging responses from all participants within the specific item. Abbreviation: GCM, group concept mapping.

the 0 to 10 NRS. Descriptive data for the domain averages are reported in Table 3 .

\section{Pattern Matching}

The clinicians versus patients importance pattern matching found the ratings by domain were similar, with the clinician and patient importance ratings being highly correlated ( $r=0.82$ ) (Figure 4). The hierarchical ordering of the average domain ratings was similar, with Prevent \& Cure and Activities of Daily Living domains rated in the top 3 in importance by both clinicians and patients. The Safe $\&$ Effective domain was rated in the top 3 by clinicians, whereas the Address Symptoms domain was rated in the top 3 by patients.

\section{Scatter Plots}

For brevity, 2 scatterplots comparing statements within the Address Symptoms and Activities of Daily Living domain are examined (Figures 5 and 6). Five statements within the Address Symptoms domain (Figure 5) were rated on importance above the respective domain mean by both clinicians and patients: \#16 "Restore sensation or feeling," \#18 “Help preserve cognition," \#20 "Relieve the symptoms to make one's daily routine easier," \#28 "Help with memory issues," and \#64 "Treat pain associated with multiple sclerosis conveniently and safely." Three statements were rated below their respective domain mean by both clinicians and patients: \#29 "Include treatment for insomnia," \#49 “Take away shaking," and \#60 "Include 
A

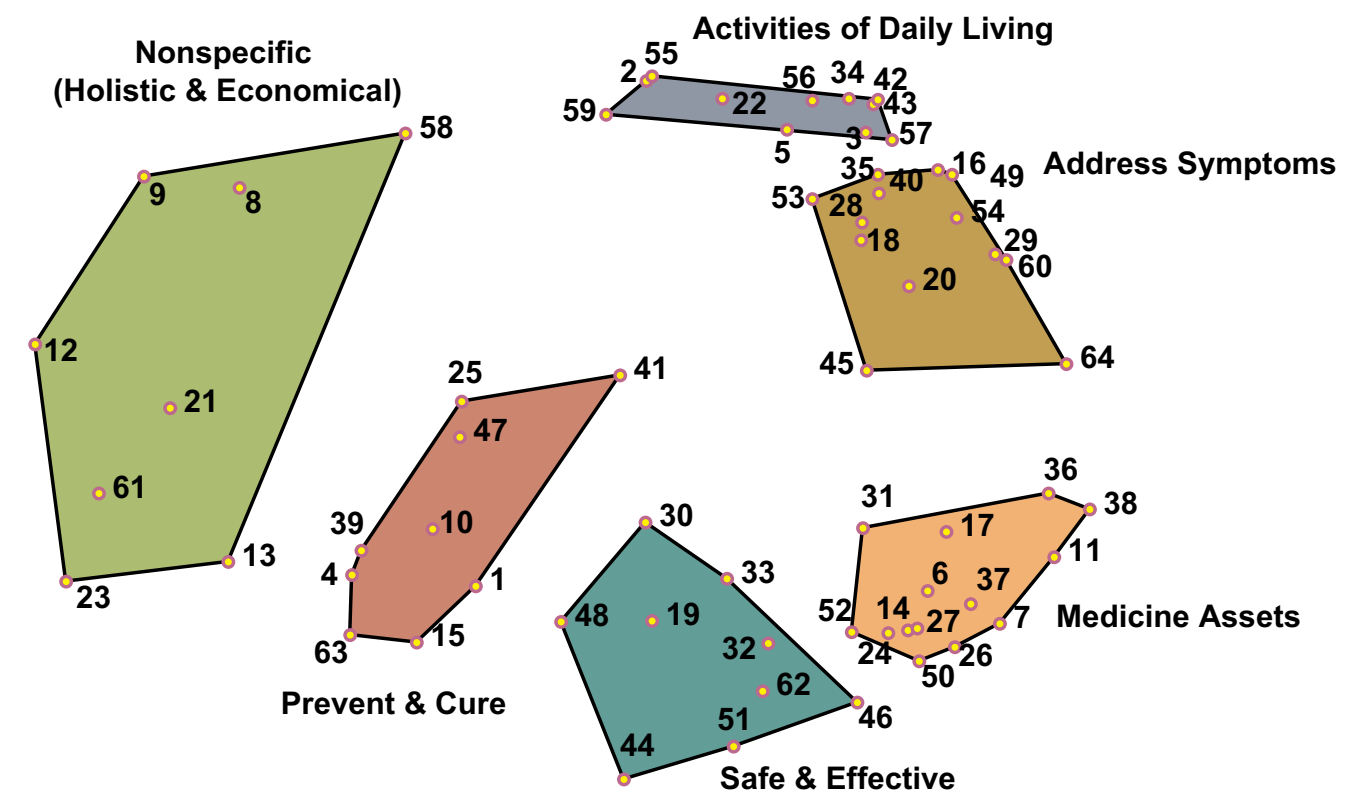

B

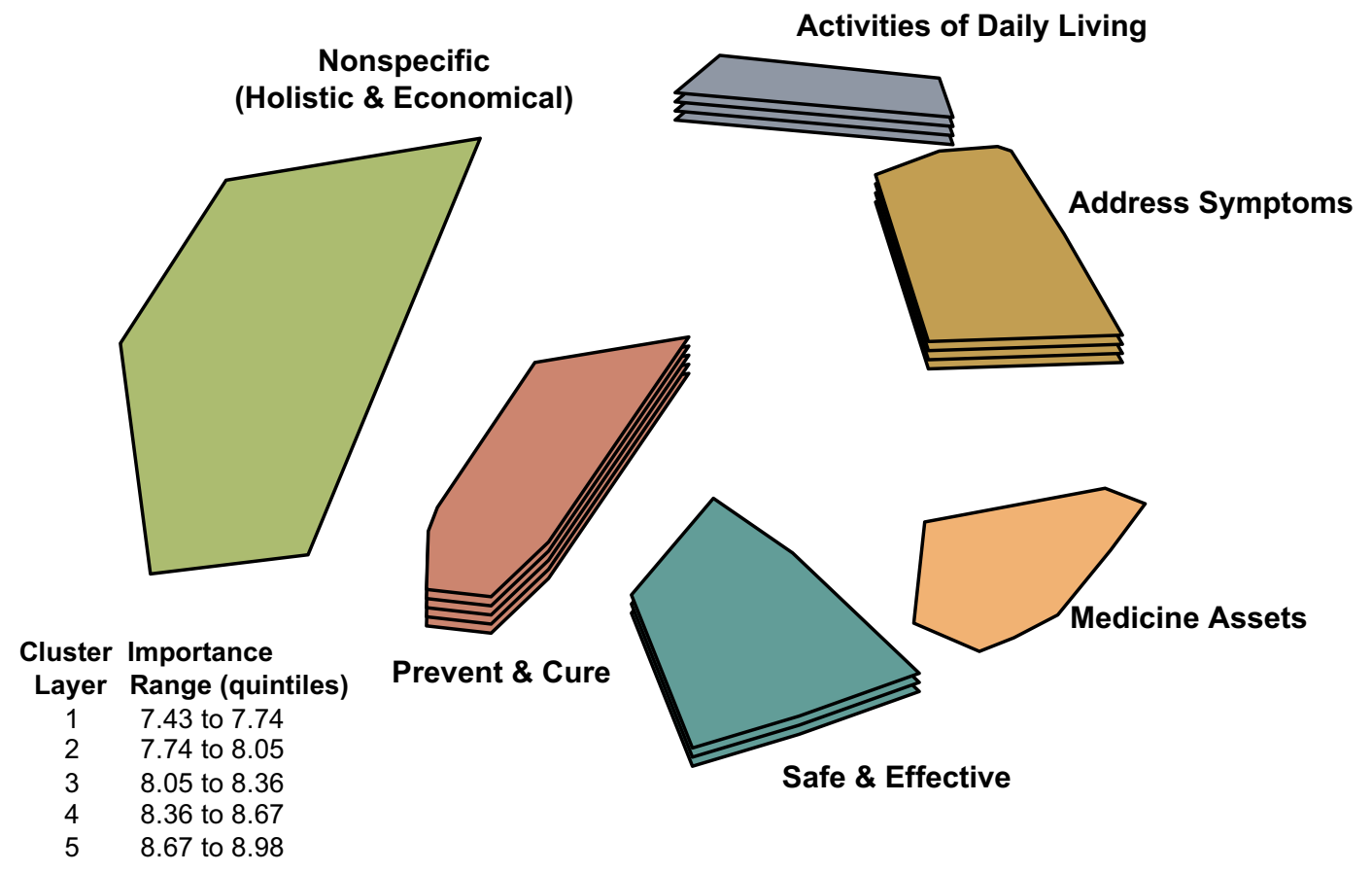

Figure 3 Cluster mapping of participant sorts and ratings. (A) Point cluster map; (B) cluster-ratings map. 


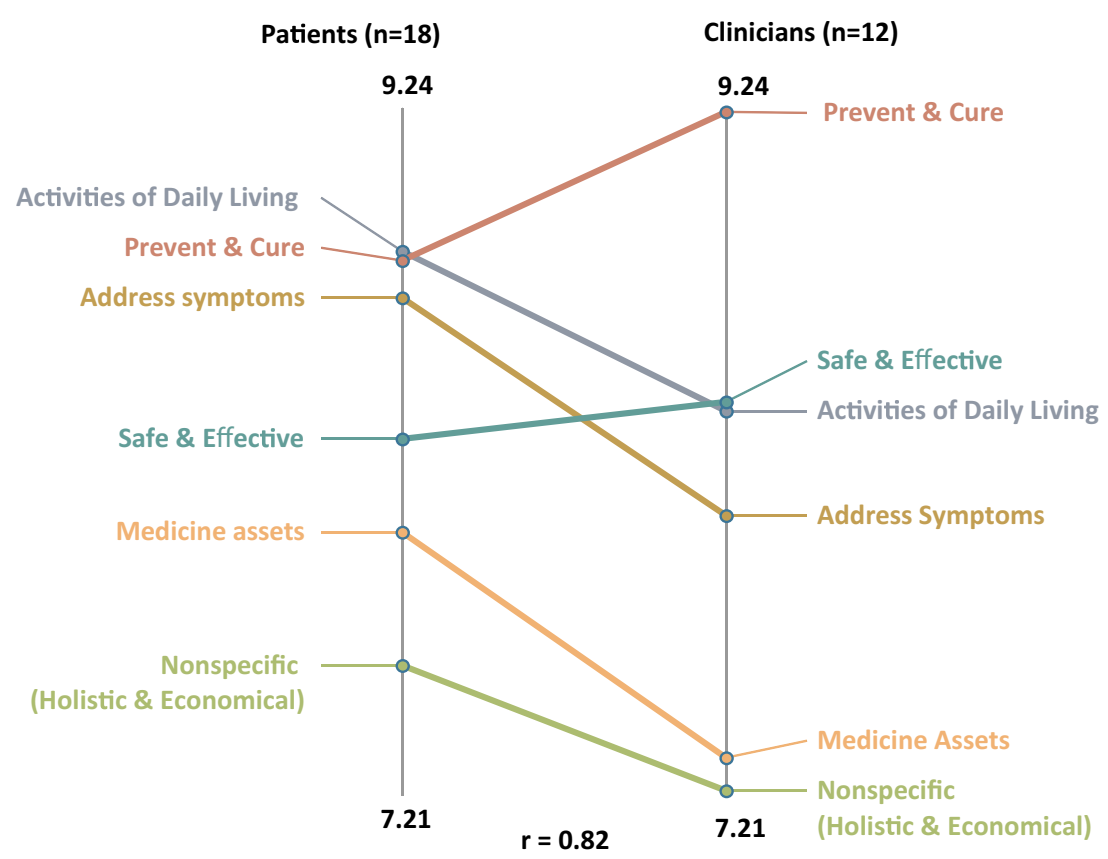

Figure 4 Pattern matching of patient and clinician importance ratings by domain.

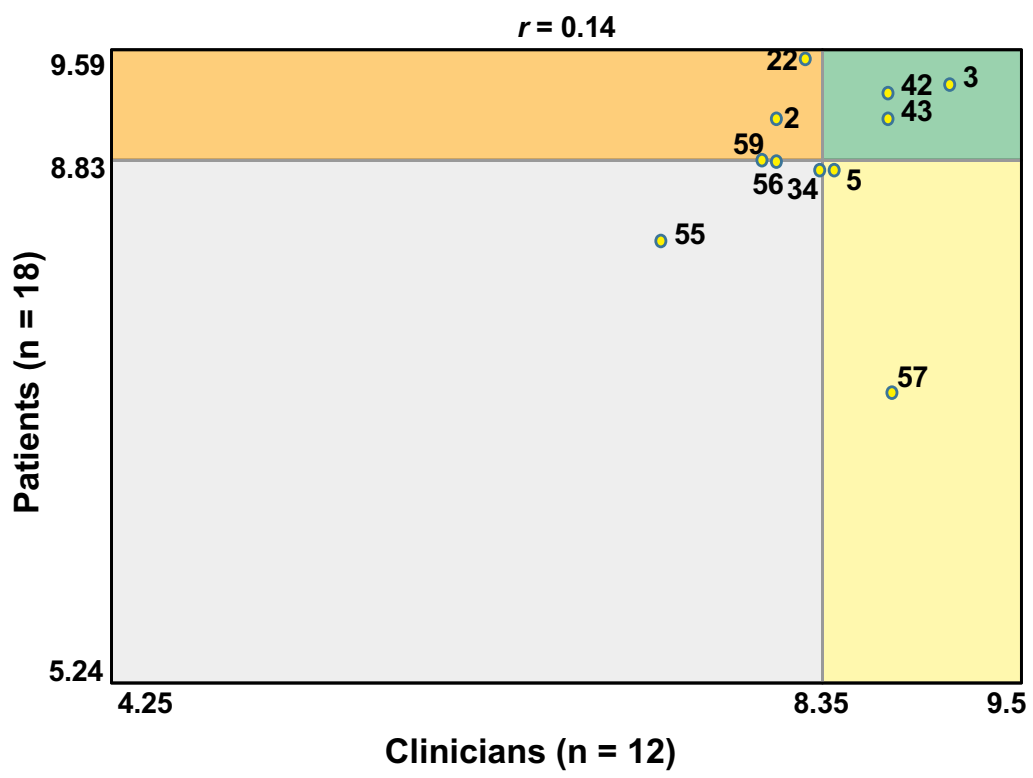

3. Improve motor function

42. Improve balance

43. Improve cognitive function

5. Help with visual impairment

57. Help improve gait

2. Help physical daily functioning for a more active social life

22. Help physical function

59. Provide more sustained energy to return to high functioning

34. Improve ambulation (walking) conveniently without harmful side effects

55. Focus on physical activity

56. Address fatigue

Figure 5 Address Symptoms domain scatterplot comparing patient and clinician mean ratings of importance by statement. The upper right quadrant (green) indicates statements above the mean for both patients and clinicians. The lower left quadrant (white) indicates statements below the mean for both patients and clinicians. The opposite quadrants indicate statements above the mean for patients/below the mean for clinicians (orange) and above the mean for clinicians/below the mean for patients (yellow).

dizziness treatment to reduce spinning." Notably, \#29 "Include treatment for insomnia" was rated relatively far below the mean by both patients and clinicians.

As shown in Figure 6, on the Activities of Daily Living domain, the patients' average importance rating was somewhat higher than the clinicians' average importance rating. The scatter plot representing the individual statement importance ratings in the Activities of Daily Living domain stratified by patients and clinicians showed that 3 statements were rated above the mean by both clinicians 


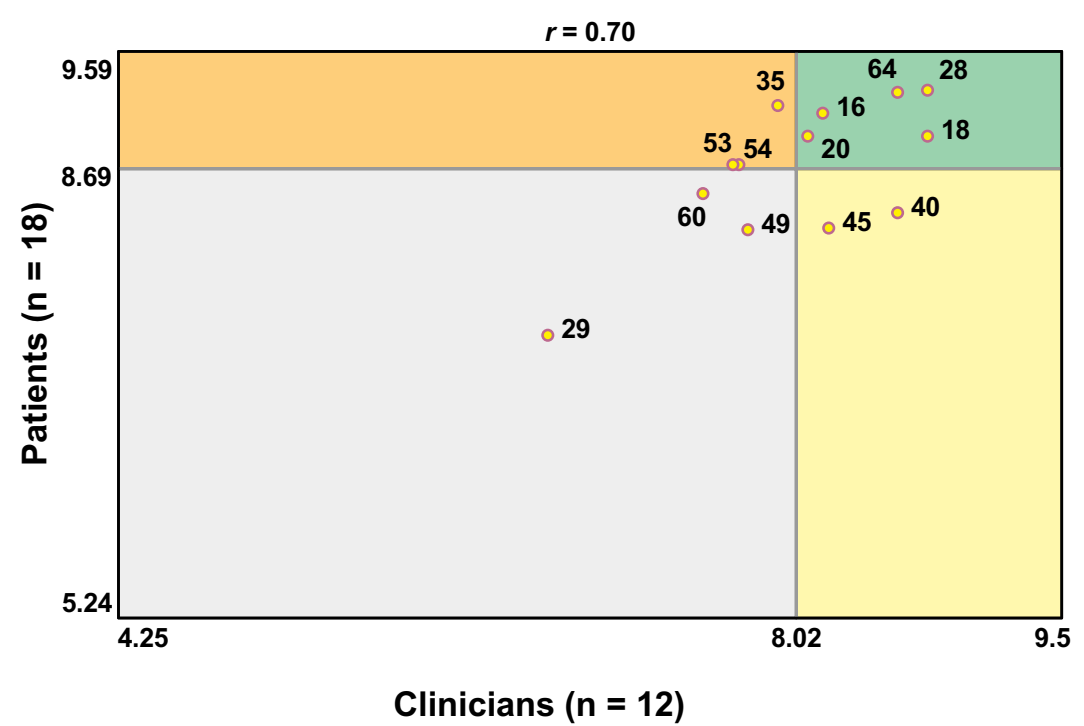
16. Restore sensation or feeling
18. Help preserve cognition
20. Relieve the symptoms to make one's daily routine easier
28. Help with memory issues
64. Treat pain associated with multiple sclerosis conveniently and safely
40. Effectively treat bladder problems
45. Be one that patients notice an improvement in their function when taking it
35. Improve short term memory
53. Provide a cure for tingling
54. Treat spasticity (stiffness, tightness, cramping, spasms) with medication
29. Include treatment for insomnia
49. Take away shaking
60. Include dizziness treatment to reduce spinning

Figure 6 Activities of Daily Living domain scatterplot comparing patient and clinician mean ratings of importance by statement. The upper right quadrant (green) indicates statements above the mean for both patients and clinicians. The lower left quadrant (white) indicates statements below the mean for both patients and clinicians. The opposite quadrants indicate statements above the mean for patients/below the mean for clinicians (orange) and above the mean for clinicians/below the mean for patients (yellow).

and patients: \#3 "Improve motor function," \#42 "Improve balance," and \#43 "Improve cognitive function." The 3 statements rated below the mean by both clinicians and patients were \#34 "Improve ambulation (walking) conveniently without harmful side effects," \#55 "Focus on physical activity," and \#56 “Address fatigue." Notably, \#57 "Help improve gait" was rated relatively far below the mean by patients but rated above the mean by clinicians.

\section{Discussion}

The statements generated by patients and clinicians regarding the ideal treatment for MS were broadly representative of previously identified symptoms and impacts, including balance, cognitive function, tremors, tingling, and fatigue. ${ }^{4}$ In addition, statements were provided that spoke to the availability of certain aspects of treatment, such as "inexpensive and/or covered by health insurance," "have minimal or no side effects," and "would enable the ability to lead a normal, healthy lifestyle." The statements were sorted by patients and clinicians, resulting in 6 domains with statements of similar content within each.

Each domain had unique characteristics. The top-rated domain, Prevent \& Cure, focused specifically on desired treatments that reverse prior deficits, stabilize progression, and protect the CNS. The Activities of Daily Living domain consisted of desire for improvements, or help, with overall function - cognitive function, physical function, ambulation/walking/gait - and visual impairment, fatigue, and desiring a more active social life. Address Symptoms had a more symptom-focused theme, with memory issues, preserve cognition, treating pain, restore sensation or feeling, along with mention of bladder problems and treatment for insomnia. A domain specifically about safety and efficacy emerged in which participants listed concepts of increasing the percentage of relapse-free patients, reducing side effects of cancer, heart problems, malignancy, and progressive multifocal leukoencephalopathy (PML). Reduced monitoring demands and efficacy decline were also listed. Medicine Assets domain had similar themes to Safety \& Efficacy domain, with mention of side effects and PML; however, treating pain without sedation and efficacy for fatigue were specifically noted. The final domain, Nonspecific (Holistic $\&$ Economical) was the lowest rated domain overall and presented the 2 themes of desire for a holistic approach to their MS, including yoga, massage, or acupuncture in their care, as well as financial and reimbursement considerations.

There was a high level of agreement between clinicians and patients in the importance ratings they gave to the individual statements and their domains overall. This indicates that patients and clinicians are well aligned with concepts for optimal patient outcomes. Although there were some differences in rating levels, 
for example, clinicians rated the statements in the Prevent \& Cure domain of slightly higher importance than did patients, whereas patients rated the statements in the Activities of Daily Living domain slightly higher than did clinicians, these differences do not indicate a large divergence between those treating patients or those with MS.

In support of previous studies of MS patient outcomes, ${ }^{7}$ our results indicated concepts of patient interest outside of the MS key symptoms of fatigue, pain, tremors/spasticity, and gait. Notably, 4 of the concepts generated by patients and clinicians were related to cognitive impairment (statements \#18, \#28, \#35, and \#43). Examination of the ratings for these cognition statements showed that, with the exception of 1 statement, "Improve short term memory," all were rated above the mean in importance by both clinicians and patients, and even this statement was rated very close to the mean by patients. Visual impairment (\#5) was included in the Activities of Daily Living domain, just slightly below the mean for clinicians. In the Address Symptoms domain, bladder problems (\#40) and treatment for insomnia (\#29) were included. Bladder problems was rated highly by patients and relatively highly by clinicians; however, treatment for insomnia rated in the midrange of the scale for both patients and clinicians.

While the goal of this study was to elicit concepts of importance for relapsing-remitting MS patients, interpretation of the study findings has some limitations. The sample size of 20 patients, though small, is generally suitable to reach saturation of concepts in a qualitative exercise. The inclusion of only $12 \mathrm{MS}$ specialists is based on clinicians being queried about opinions based on their technical expertise, rather than personal opinions, thus being a more homogeneous sample. Comparisons between patients and clinicians are merely for descriptive purposes in this manuscript. Further work in this area would require debriefing studies to support the model, in addition to further comparisons of statements including other response frameworks, such as ranking of priorities or best to worst scaling, to further probe patient priorities. The strengths of this methodology included participant generation of all statements, with minimal editing from the research team and a cluster solution based on the individual participant sorts rather than a researcher-based coding schema. The ratings information provides insights into potential areas of disparity between the patients and clinicians, and as shown, minimal differences were discovered within the symptom- and impact-focused domains.

\section{Conclusion}

Patient expectations and priorities for treatment outcomes should be considered during treatment decisions by clinicians as well as during the research and development of new treatments. Our research highlights a high level of agreement between patients and clinicians on the most important concepts related to the prevention and cure, as well as the treatment, of MS, including concepts relating to cognitive function, physical and emotional function, and activities of daily living, which are considered important by both clinicians and patients. This comprehensive concept model for the RRMS patient experience can be used to further develop patientcentered outcome measures in the treatment of MS.

\section{Abbreviations}

2-D, 2-dimensional; CNS, central nervous system; GCM, group concept mapping; HCA, hierarchical cluster analysis; MDS, multidimensional scaling; MS, multiple sclerosis; NRS, numeric rating scale; PRO, patient-reported outcomes; PML, progressive multifocal leukoencephalopathy; RRMS, relapsing-remitting multiple sclerosis.

\section{Data Sharing Statement}

BMS policy on data sharing may be found at https://www. bms.com/researchers-and-partners/independent-research/ data-sharing-request-process.html.

\section{Acknowledgments}

GCM analytic support was provided by Scott Rosas of Concept Systems Inc (Albany, OR, USA). Third-party medical writing assistance for this manuscript was provided by Clinical Outcomes Solutions, Chicago, IL, USA, and editorial support was provided by Peloton Advantage, LLC (Parsippany, NJ, USA), an OPEN Health company, both sponsored by Bristol Myers Squibb (Princeton, NJ, USA). The authors, however, directed and are fully responsible for all content and editorial decisions for this manuscript.

\section{Author Contributions}

BAS, SK, AH, HD, and RM contributed to the conception, analysis, interpretation, and revised manuscript content. TP contributed to interpretation and revising content. All authors read and approved the final manuscript. All authors made substantial contributions to conception and design, acquisition of data, or analysis and interpretation of data; took part 
in drafting the article or revising it critically for important intellectual content; agreed to submit to the current journal; gave final approval of the version to be published; and agree to be accountable for all aspects of the work.

\section{Funding}

This study was supported by Bristol Myers Squibb. Bristol Myers Squibb was involved in the design and conduct of the study; collection, management, analysis, and interpretation of the data; and preparation, review, and approval of the manuscript.

\section{Disclosure}

BAS: Speaker and consulting fees from AbbVie, Alexion, Bayer, Biogen, Celgene (Bristol Myers Squibb), EMD Serono, Genentech, Greenwich Biosciences, Novartis, Roche, Sanofi Genzyme, Teva, and TG Therapeutics. Research support from AbbVie, Alkermes, Biogen, MedImmune, Novartis, Sanofi Genzyme, and Roche. AH, HD and SK: Employed at Clinical Outcomes Solutions. RM and TP: Employed at Bristol Myers Squibb currently or at the time the study was conducted. The authors report no other conflicts of interest in this work.

\section{References}

1. Tullman MJ. Overview of the epidemiology, diagnosis, and disease progression associated with multiple sclerosis. Am J Manag Care. 2013;19(2 Suppl):S15-S20.

2. National Multiple Sclerosis Society. Fatigue: what you should know. A guide for people with MS. Consumer Guide to Clinical Practice Guidelines. 2019; Availbale from: https:/www.nationalmssociety.org/ NationalMSSociety/media/MSNationalFiles/Brochures/Brochure-

Fatigue-What-You-Should-Know.pdf. Accessed September 24, 2020.
3. Wallin MT, Culpepper WJ, Campbell JD, et al. The prevalence of MS in the United States: a population-based estimate using health claims data. Neurology. 2019;92(10):e1029-e1040. doi: 10.1212/WNL.0000 000000007035

4. Zwibel HL, Smrtka J. Improving quality of life in multiple sclerosis: an unmet need. Am J Manag Care. 2011;17(Suppl 5):S139-S145.

5. Comi G, Radaelli M, Soelberg Sorensen P. Evolving concepts in the treatment of relapsing multiple sclerosis. Lancet. 2017;389 (10076):1347-1356. doi: 10.1016/S0140-6736(16)32388-1

6. Martin S, Olayinka-Amao O, Henke C, Kamudoni P, Gnanasakthy A. Conceptual model and instrument review in multiple sclerosis [abstract PND53]. Value Health. 2017;20(9):PA726-PA727. doi: 10.1016/j.jval.2017.08.1967

7. Col NF, Solomon AJ, Springmann V, et al. Whose preferences matter? A patient-centered approach for eliciting treatment goals. Med Decis Making. 2018;38(1):44-55. doi: 10.1177/0272989X17 724434

8. Eskyte I, Manzano A, Pepper G, et al. Understanding treatment decisions from the perspective of people with relapsing remitting multiple sclerosis: a critical interpretive synthesis. Mult Scler Relat Disord. 2019;27:370-377. doi: 10.1016/j.msard.2018.11.016

9. Webb EJD, Meads D, Eskyte I, et al. A systematic review of discrete-choice experiments and conjoint analysis studies in people with multiple sclerosis. Patient. 2018;11(4):391-402. doi: 10.1007/ s40271-017-0296-y

10. Rising KL, LaNoue M, Gentsch AT, et al. The power of the group: comparison of interviews and group concept mapping for identifying patient-important outcomes of care. BMC Med Res Methodol. 2019;19(1):7. doi: 10.1186/s12874-018-0656-x

11. Rosas SR, Camphausen LC. The use of concept mapping for scale development and validation in evaluation. Eval Program Plann. 2007;30(2):125-135. doi: 10.1016/j.evalprogplan.2007.01.003

12. Concept Systems Incorporated. Concept Systems global MAX ${ }^{\mathrm{TM}}$; 2019. Available from: www.conceptsystems.com/home. Accessed April 12, 2021.

13. Kane M, Trochim WMK. Concept Mapping for Planning and Evaluation. Vol 50. In: Bickman L, Rog DJ, editors. Thousand Oaks, CA: Sage Publications, Inc.; 2007. doi:10.4135/ 9781412983730

14. Kane M, Rosas S. Conversations About Group Concept Mapping: Applications, Examples, and Enhancements. Los Angeles, CA: Sage Publications, Inc.; 2018.

15. Davison M. Multidimensional Scaling. New York: Wiley; 1983.
Patient Preference and Adherence

\section{Publish your work in this journal}

Patient Preference and Adherence is an international, peer-reviewed, open access journal that focusing on the growing importance of patient preference and adherence throughout the therapeutic continuum. Patient satisfaction, acceptability, quality of life, compliance, persistence and their role in developing new therapeutic modalities and compounds to optimize clinical outcomes for existing disease states are major areas of interest for the journal. This journal has been accepted for indexing on PubMed Central. The manuscript management system is completely online and includes a very quick and fair peer-review system, which is all easy to use. Visit http:// www.dovepress.com/testimonials.php to read real quotes from published authors. 